\title{
Totally Laparoscopic vs Open Transhiatal Esophagectomy: Our Experience in 93 Patients
}

\author{
Ali Jangjoo ${ }^{1}$, Sadjad Noorshafiee ${ }^{2}$, Ehsan Alaei ${ }^{3}$, Yasaman Navari ${ }^{4}$, Mahdi Jabbari Nooghabi ${ }^{5}$
}

\begin{abstract}
Introduction: The incidence of esophageal cancer has been increasing in the last decade. Different types of treatments are available, including minimally invasive esophagectomy (MIE). The aim of this study was to compare the early outcomes of the open vs totally laparoscopic transhiatal esophagectomy.

Materials and methods: This case-control study was conducted between May 2012 and January 2014. Patients with esophageal cancer who presented to Imam Reza Hospital, Mashhad, Iran, were assessed and their eligibility for the surgery type was investigated.

Results: Ninety-three esophagectomies performed. The open group comprised 57 patients and the laparoscopic group consisted of 36 patients. Mortality occurred in three patients in the open group and seven patients in the laparoscopic group $(p<0.05)$. Chylothorax happened in four patients in the open group and only in one patient in the laparoscopic, which showed no significant difference. The mean operating time was $75 \pm 16$ minutes in the open group and $125 \pm 25$ minutes in the laparoscopic group $(p<0.05)$.

Conclusion: Minimally invasive transhiatal esophagectomy is an available option for treatment of esophageal cancer, but our results should be interpreted with caution due to low sample size and our primary experience in patient selection.

Keywords: Esophageal cancer, Esophagectomy, Laparoscopy, Transhiatal.

World Journal of Laparoscopic Surgery (2019): 10.5005/jp-journals-10033-1370
\end{abstract}

\section{INTRODUCTION}

The incidence of esophageal cancer has been increasing in the last decades. ${ }^{1}$ In the United States, 17,000 new cases are diagnosed and 15,000 cases die annually. ${ }^{2}$ In our region in the north of Iran, this disease is endemic with a prevalence rate of $180 / 1,00,000$ population. The mean age of the patients is 60 years. Different types of therapy are available for this cancer, but there is a general agreement that surgery could be the best option. ${ }^{1-10}$ Surgical treatments consist of different types of methods that are used today. Most types of current surgical approaches are invasive and harsh and have multiple complications especially respiratory one. Today, minimally invasive methods, including laparoscopy, have progressively improved. ${ }^{5,10,11}$ The aim of this study was to compare the early outcomes of the open vs totally laparoscopic transhiatal esophagectomy.

\section{Materials and Methods}

This pilot case-control study was conducted between May 2012 and January 2014 after obtaining the approval of the Ethics Committee of Mashhad University of Medical Sciences, Mashhad, Iran. Study population included 93 patients, after excluding patients with missing data; the study participants consisted of patients who presented with esophageal cancer to Imam Reza Hospital and were candidates for surgery after preoperative workup and approval of their eligibility by a multidisciplinary team. Patients with cervical esophagus tumor, prior open upper abdominal surgeries, and tumor higher than carina level were excluded from the study. All patients underwent neoadjuvant chemoradiotherapy at least 4 weeks before surgery. The procedures were described and an informed consent was obtained from all patients. They were randomly assigned to two groups, namely, open and laparoscopy groups. Patients underwent either open or laparoscopic transhiatal

\footnotetext{
1,3Surgical Oncology Research Center, Mashhad University of Medical Sciences, Mashhad, Iran

${ }^{2,4}$ Endoscopic and Minimally Invasive Surgery Research Center, Mashhad University of Medical Sciences, Mashhad, Iran

${ }^{5}$ Department of Statistics, Faculty of Mathematical Sciences, Ferdowsi
} University of Mashhad, Mashhad, Iran

Corresponding Author: Sadjad Noorshafiee, Endoscopic and Minimally Invasive Surgery Research Center, Mashhad University of Medical Sciences, Mashhad, Iran, Phone: +98 513-8402972, e-mail: emis@mums.ac.ir

How to cite this article: Jangjoo A, Noorshafiee S, Alaei E, et al. Totally Laparoscopic vs Open Transhiatal Esophagectomy: Our Experience in 93 Patients. World J Lap Surg 2019;12(2):56-58.

Source of support: Research Department of Mashhad University of Medical Sciences (ID: 940066)

Conflict of interest: None

esophagectomy. The data concerning the type of operation, type of conduit, pylorus drainage procedures, intraoperative complications, chest tube insertion and amount and type of drained fluid, postoperative complications, operative time, and the need for reoperation were recorded. Then the data were analyzed using SPSS version 16 with Fisher's exact test and Pearson's Chi-square test. $p$ value $<0.05$ was set as statistically significant.

\section{Surgical Technique}

Patients were operated on under general anesthesia in supine position with legs apart. The surgeon stands between the patient's legs. The peritoneal cavity was explored for metastasis or any finding that precludes safe surgery via $10 \mathrm{~mm}$ infraumbilical port. Another $10 \mathrm{~mm}$ port was inserted for ligature device $(10 \times 35$; Covedien, USA) for dissection in left subcostal area in the region

(c) The Author(s). 2019Open Access This article is distributed under the terms of the Creative Commons Attribution 4.0 International License (https://creativecommons. org/licenses/by-nc/4.0/), which permits unrestricted use, distribution, and non-commercial reproduction in any medium, provided you give appropriate credit to the original author(s) and the source, provide a link to the Creative Commons license, and indicate if changes were made. The Creative Commons Public Domain Dedication waiver (http://creativecommons.org/publicdomain/zero/1.0/) applies to the data made available in this article, unless otherwise stated. 
nearest to the neck. The $5 \mathrm{~mm}$ ports were placed in subxiphoid for liver retractor, right subcostal region in midclavicular line, for the left hand of the surgeon, and left subcostal anterior axillary line for the assistant. Then the left lobe of the liver was retracted and dissection began by dividing the phrenoesophageal ligament. The esophagus was dissected from the adjacent crura. The abdominal esophagus was handled with a surgical tape encircling it for different maneuvers for dissection of the mediastinal part of the esophagus. Then the esophagus was dissected up as high as possible to the neck, under the direct vision of the scope. Thereafter, gastro lysis was performed by preserving the right gastric and right gastroepiploic arcades using a $10 \times 35$ ligature. Kocher maneuver and pyloroplasty were not performed. After dissecting the left gastric artery lymph nodes, the gastroesophageal junction was stapled and divided to make a conduit. Then a suture was tied between the conduit and the esophagus to pull the stomach up through the neck. An oblique incision was made parallel and anterior to the left sternocleidomastoid muscle and the cervical esophagus was explored, dissected, and brought to the incision. In this step, care was taken to avoid recurrent laryngeal nerve injury. The stomach was brought up to the neck via esophagus traction and the suture between them was cut and a hand-sewn singlelayer end-to-side esophagogastric anastomosis was performed. No nasogastric tube was used. Then the anastomosis was drawn back to the neck and the incision was closed with simple nylon sutures. No feeding jejunostomy was used. If required, the chest tube was inserted at the end of the operation. All patients were transferred to the intensive care unit.

The open procedure was performed in the same manner but via midline laparotomy.

\section{Statistical Analysis}

The data concerning the type of operation, type of conduit, pylorus drainage procedures, intraoperative complications, chest tube insertion, and amount and type of drained fluid, postoperative complications, operative time, and need for reoperation were recorded. Then the data were analyzed using SPSS version 16 with Fisher's exact test and Peterson's Chi-square test. $p$ value $<0.05$ was set as statistically significant.

\section{ResUlts}

Totally, 93 patients were enrolled in the study. Of the 93 patients, 57 had open procedure and 36 had laparoscopic esophagectomy. The mean age was $60 \pm 11$ and male to female (M:F) ratio was 1.5:2 in open group and the mean age was $57 \pm 15$ and M:F ratio was 1.4:2 in laparoscopic group without significant difference. Mortality occurred in $3(5.3 \%)$ patients in the open group and $7(19.4 \%)$ patients in the laparoscopic group $(p=0.037)$. Four $(7 \%)$ patients had chylothorax in the open group and 1 (2.8\%) patient in laparoscopic group ( $p=0.354)$. Other morbidities happened in both groups but without statistically significant difference, consisting of one recurrent laryngeal nerve palsy in open group and two case of neck fistula in laparoscopic group. Fifteen (26.3\%) patients had chest tube in open group and 8 (22.2\%) patients in laparoscopic group ( $p=0.425)$. Three categories of chest tube drainage were considered, namely, more than $400 \mathrm{cc}$, less than $400 \mathrm{cc}$, and no drainage. In the open group, 10 patients had more than $400 \mathrm{cc}(17.5 \%), 4$ patients had less than $400 \mathrm{cc}(7 \%)$, and $43 \mathrm{had}$ no drainage. In the laparoscopic group, 3 patients had more than
$400 \mathrm{cc}$ and 33 (91.7\%) patients had no drainage. The two groups showed no significant difference in term of the drainage volume $(p=0.087)$.

Regarding mortality and morbidity, no intraoperative death, and no major bleeding occurred; packed cell transfusion was also not required.

The mean operative time was $75 \pm 16$ minutes in the open group and $125 \pm 25$ minutes in the laparoscopic group $(p<0.05)$.

Two laparoscopic procedures were converted to open, i.e., one patient due to tumor invasion to aorta and one due to invasion to carina. In another case, the lack of vision and inability to dissect the esophagus led to open surgery. Hospital staying was $8 \pm 1.7$ days in open group and $7 \pm 1.9$ days in laparoscopic group $(p>0.05)$.

\section{Discussion}

According to the literature, minimally invasive esophagectomy (MIE) is usually a combination of laparoscopy, thoracoscopy, laparotomy or thoracotomy, with or without cervical anastomosis. ${ }^{1-3}$ In the study by Luketich et al. ${ }^{1}$ which was performed on more than 1,000 MIEs, two groups were compared in terms of laparoscopicthoracoscopic MIE (Ivor Lewis MIE) and thoracoscopy-laparoscopy neck anastomosis (McKeown MIE or neck MIE). In the view of the complications and mortality, the total 30-day in-hospital mortality in both the groups was $1.68 \%$. In our study, a $10 \%$ mortality rate was observed in hospital, with no out-hospital mortality in the 30-day period after surgery. Their study indicated no difference in mortality in two MIE groups, but in our study the mortality was statistically higher in the laparoscopic group. This can be partially attributed to the learning curve issues with minimally invasive approaches and poor patient selection. We should mention that their technique is completely different. In another study by Meng et al., ${ }^{2}$ open transhiatal esophagectomy was compared to MIE using thoracoscopic combined with minilaparotomy in 183 patients. Total mortality was five patients, which was lower than ours. Postoperative complications including anastomosis leakage ( 8 vs 2 in ours) and recurrent laryngeal nerve injury ( 8 vs 2 in ours) were similar in both groups. They reported chylothorax in $7(3 \%)$ patients, but in our study it occurred in $5(7 \%)$ patients. But these complications were the same in both groups in their study. Another study ${ }^{12}$ compared the results of open vs laparoscopic transhiatal esophagectomy. The only difference in laparoscopic technique was abdominal phase of the operation which was performed in epigastrium with a $7-\mathrm{cm}$ minilaparotomy. Just like our study, no pyloroplasty was performed in the laparoscopic group; but unlike our study, only 36 patients had neoadjuvant chemoradiotherapy. They had 9 (18\%) conversions to open surgery, but we had 2 conversions. Similar to our study, laparoscopic group had longer operative time (300 minutes). No mortality was reported in MIE group, but one was reported in the in open group. In our study, three patients who underwent open surgery died and seven patients in the laparoscopy group. This difference may be due to patient selection or tumor location. Compared with our study, they had more morbidity, recurrent laryngeal nerve palsy ( 8 vs 1 in our study) and neck fistula (7 vs 2 in our study), but they reported less chylothorax (3 vs 5 in our study). In another study by Rodham et al., ${ }^{13}$ patients were studied in view of hospital stays. They reviewed 24 studies and concluded that patients underwent MIE by any method had lower hospital stay (mean of 3 days). In our study, the two groups showed no statistically significant difference in terms of hospital stay. 
In an review article by Herbella et al., ${ }^{14}$ different types of MIE and conventional open surgery were compared and finally they concluded that MIE has less mortality and morbidity with the same oncologic results, but in our study mortality was higher and morbidity was lower in MIE.

In a study by Khithani et al., ${ }^{15}$ no mortality was observed after performing MIE using minilaparotomy. Interestingly, the major morbidity was pneumonia in 8 (24\%) patients compared with 0 in our study. But they had no cervical leak. A study by Lee et al. ${ }^{16}$ demonstrated that the only difference between MIE and open surgery is the abdominal operative time $(90 \pm 27.6$ minutes in the laparoscopic group vs $162 \pm 97.3$ minutes in the open group; $p<0.001)$, which is consistent with our finding ( $76 \pm 15$ minutes vs $125 \pm 25$ minutes, respectively, in laparoscopic and open group; $p<0.05)$. They had a patient with pneumonia. Their mortality was 3 in MIE group and 2 in the open group. Their results were better than ours in terms of mortality. In another study by Gao et al., ${ }^{17}$ they compared the MIE (thoracoscopy, minilaparotomy, and cervical anastomosis) to open surgery. The operative time was higher in MIE group. They observed more pulmonary complications and anastomosis leakage in MIE group but mortality did not differ statistically in two groups.

\section{Conclusion}

In summary, previous studies demonstrated heterogeneous results, which may result from different patient selections, different techniques, and study designs. Overall, we can conclude that there are reports on MIE with more patients undergoing unique techniques and involving better clarified patient selection to prevent mortality, morbidity, and heterogeneity of results.

Preoperative location of tumor should be defined by barium swallow and computed tomography scan. Endoscopic ultrasonography for clarifying the T stage of tumor is advocated for all patients. We also advocate using total laparoscopic transhiatal esophagectomy in tumors distal to carina level and early stage tumors. Preoperative chemoradiotherapy may decrease the bulk of tumor, thus optimizing the handling of tumor in the narrow space of mediastinum though it may obscure the dissection planes.

\section{Compliance with Ethical Standards Informed Consent}

Informed consent was obtained from all individual participants included in the study.

\section{Ethical Approval}

All procedures performed in studies involving human participants were in accordance with the ethical standards of ethics Committee of Mashhad University of Medical Sciences, Mashhad, Iran.

\section{Acknowledgments}

The results described in this paper formed part of a thesis submitted by the third author for a postgraduate degree in surgery. The authors hereby wish to thank the Research Department of Mashhad University of Medical Sciences for its support and approval of the proposal number 940066 and Ms. S Beigoli and Ms. M Hassanpour for their kind assistance in preparing the paper.

\section{References}

1. Luketich JD, Pennathur $A$, Awais $\mathrm{O}$, et al. Outcomes after minimally invasive esophagectomy: review of over 1000 patients. Ann Surg 2012;256(1):95-103. DOI: 10.1097/SLA.0b013e3182590603.

2. Meng F, Li Y, Ma H, et al. Comparison of outcomes of open and minimally invasive esophagectomy in 183 patients with cancer. J Thorac Dis 2014;6(9):1218-1224. DOI: 10.3978/j.issn.20721439.2014.07.20.

3. Biere SS, Maas KW, Bonavina L, et al. Traditional invasive vs. minimally invasive esophagectomy: a multi-center, randomized trial (TIME-trial). BMC Surg 2011;11:2. DOI: 10.1186/1471-2482-11-2.

4. Puntambekar $\mathrm{S}$, Kenawadekar R, Pandit A, et al. Minimally invasive esophagectomy in the elderly. Indian J Surg Oncol 2013;4(4):326-331. DOI: 10.1007/s13193-013-0263-6.

5. Santin BJ, Price P. Laparoscopic transhiatal esophagectomy at a lowvolume center. JSLS 2011;15(1):41-46. DOI: 10.4293/108680811X1302 2985131138.

6. Smithers BM, Gotley DC, Martin I, et al. Comparison of the outcomes between open and minimally invasive esophagectomy. Ann Surg 2007;245(2):232-240. DOI: 10.1097/01.sla.0000225093.58071.c6.

7. Avery KN, Metcalfe C, Berrisford R, et al. The feasibility of a randomized controlled trial of esophagectomy for esophageal cancer-the ROMIO (Randomized Oesophagectomy: Minimally Invasive or Open) study: protocol for a randomized controlled trial. Trials 2014;15:200. DOI: $10.1186 / 1745-6215-15-200$.

8. Beigoli S, Sharifi Rad A, Askari A, et al. Isothermal titration calorimetry and stopped flow circular dichroism investigations of the interaction between lomefloxacin and human serum albumin in the presence of amino acids. J Biomol Struct Dyn 2019;37(9):2265-2282. DOI: 10.1080/07391102.2018.1491421.

9. Briez N, Piessen G, Bonnetain F, et al. Open versus laparoscopicallyassisted oesophagectomy for cancer: a multicentre randomised controlled phase III trial—the MIRO trial. BMC Cancer 2011;11:310. DOI: 10.1186/1471-2407-11-310.

10. Luketich JD, Alvelo-Rivera M, Buenaventura PO, et al. Minimally invasive esophagectomy: outcomes in 222 patients. Ann Surg 2003;238(4):486-494; discussion 94-95. DOI: 10.1097/01. sla.0000089858.40725.68.

11. Wallner G, Zgodzinski W, Masiak-Segit W, et al. Minimally invasive surgery for esophageal cancer-benefits and controversies. Kardiochir Torakochirurgia Pol 2014;11(2):151-155. DOI: 10.5114/ kitp.2014.43842.

12. Maas KW, Biere SS, Scheepers JJ, et al. Laparoscopic versus open transhiatal esophagectomy for distal and junction cancer. Rev Esp Enferm Dig 2012;104(4):197-202. DOI: 10.4321/S113001082012000400005.

13. Rodham P, Batty JA, McElnay PJ, et al. Does minimally invasive oesophagectomy provide a benefit in hospital length of stay when compared with open oesophagectomy? Interact Cardiovasc Thorac Surg 2016;22(3):360-367. DOI: 10.1093/icvts/ivv339.

14. Herbella FA, Patti MG. Minimally invasive esophagectomy. World J Gastroenterol 2010;16(30):3811-3815. DOI: 10.3748/wjg.v16.i30.3811.

15. Khithani A, Jay J, Galanopoulos C, et al. Zero leaks with minimally invasive esophagectomy: a team-based approach. JSLS 2009;13(4):542-549. DOI: 10.4293/108680809X12589998404326.

16. Lee JW, Sung SW, Park JK, et al. Laparoscopic gastric tube formation with pyloromyotomy for reconstruction in patients with esophageal cancer. Ann Surg Treat Res 2015;89(3):17-23. DOI: 10.4174/ astr.2015.89.3.117.

17. Gao $Y$, Wang $Y$, Chen $L$, et al. Comparison of open three-field and minimally-invasive esophagectomy for esophageal cancer. Interact Cardiovasc Thorac Surg 2011;12(3):366-369. DOI: 10.1510/ icvts.2010.258632. 